\title{
G-Quadruplex Stabilizer BMVC
}

National Cancer Institute

\section{Source}

National Cancer Institute. G-Quadruplex Stabilizer BMVC. NCI Thesaurus. Code C95893.

A carbazole derivative (3,6-bis[2-(1-methylpyridinium)vinyl]carbazole diiodide) that selectively targets to the G-quadruplex DNA structure, used as a fluorescent probe for cancer cytological diagnosis and with potential antitumor activity. G-quadruplex stabilizer BMVC, preferentially uptaken by cancer cells, binds to and stabilize the telomeric Gquadruplex structure at the end of DNA; when visualized with fluorescent imaging device, BMVC emits bright fluorescent light and can be used to differentiate tumor cells from normal cells. The BMVC/G-quadruplex complexes also interfere with the activity of telomerase, which is highly active in tumor cells and plays a key role in tumorigenesis while expressed at very low levels in most somatic cells. 